\title{
Asociación entre conducta sedentaria y diabetes gestacional
}

\author{
Association between sedentary behavior and gestational \\ diabetes
}

Astrid von Oetinger Giacoman', Luz María Trujillo Gittermann²-3, Daniela García León'-2

Fecha correspondencia:

Recibido: noviembre 19 de 2019.

Revisado: noviembre 21 de 2019.

Aceptado: noviembre 21 de 2019.

Forma de citar:

von Oetinger A, Trujillo LM, Garcia

D. Asociación entre conducta

sedentaria y diabetes gestacional.

Rev CES Med 2020; 34(1): 90-91.

Open access

(C) Derecho de autor

Licencia creative commons

Ética de publicaciones

Revisión por pares

Gestión por Open Journal System

DOl: http://dx.doi.org/10.21615/

cesmedicina.34.1.9

ISSN 0120-8705

e-ISSN 2215-9177

Sobre los autores:

1. Escuela de Kinesiología,

Facultad de Salud, Universidad San

Sebastián, Santiago, Chile.

2. Escuela de Kinesiología, Facultad

Comparte

F日G $898 \mathbb{R}^{0}$ 国

\section{Estimado editor}

Teniendo en cuenta las alarmantes tasas de obesidad mundial y el aumento en la conducta sedentaria, consideramos importante determinar cómo esto podría afectar a mujeres embarazadas (1). La conducta sedentaria se define como estar por un tiempo prolongado (más de tres horas) en actividades de bajo gasto energético (1 a 1,5 MRTS), que incluyen estar sedente, acostado, viendo televisión, leer, etc., durante el tiempo de vigilia (2).

Esta conducta sedentaria aumenta el riesgo de desarrollar obesidad, cáncer, diabetes y enfermedades cardiovasculares $(3,4)$. Una revisión sistemática de 2017 determina que las embarazadas pasan más de la mitad de su tiempo en vigilia en conductas sedentarias, prácticamente el mismo tiempo que invierte la población general (5). A pesar de esto, solo existe un estudio que asocia la conducta sedentaria con la diabetes gestacional y los niveles de glucosa durante el embarazo, y por esta razón consideramos de suma relevancia referirnos al reciente artículo de Wagnild et al. de 2019 (6).

En este dicho trabajo, los autores determinan la asociación entre el tiempo de conducta sedentaria durante el embarazo y la incidencia de diabetes gestacional. Los autores midieron el tiempo sedentario (acelerómetro activPAL3), mientras que el tiempo viendo televisión fue evaluado con autoreporte. Un total de 188 mujeres embarazadas con al menos un factor de riesgo de desarrollar diabetes gestacional fueron parte de esta investigación. Las mediciones comenzaron a las 20 semanas de gestación. Entre las semanas 24 y 28 se realizó una prueba de tolerancia oral de glucosa (OGTT, por sus siglas en inglés), y se registraron los valores de glicemia en ayunas y postprandial (dos horas).

En aquellas mujeres que no desarrollaron diabetes gestacional no había asociación entre conducta sedentaria e incidencia de diabetes gestacional. En este mismo grupo los autores encuentran asociación entre conducta sedentaria y glicemia en ayuno y postprandial $(p<0,05)$.

En las mujeres que desarrollaron diabetes gestacional existe asociación entre los cambios de su conducta sedentaria y menores glicemias en ayuno y postprandial $(p<0,05)$. Además, un mayor tiempo en conducta sedentaria se asocia con mayores niveles de glicemia en ayunas $(p<0,05)$. También 
de Odontología y Salud, Universidad Diego Portales, Santiago, Chile.

3. Escuela de Kinesiología, Facultad de Ciencias de la Salud, Universidad de Las Américas, Santiago, Chile. reportan asociación entre el tiempo viendo televisión y el desarrollo de diabetes gestacional. Los autores proponen reducir la conducta sedentaria e incluir cambios de conducta sedentaria en las mujeres embarazadas, para reducir la glicemia y el riesgo de diabetes gestacional.

Aunque este estudio es el primero en abordar esta temática, consideramos que sus resultados son bastante prometedores, ya que abren posibilidades a nuevas formas de abordaje de la diabetes gestacional, mejorando la salud de las mujeres embarazadas con estrategias simples que pueden derivar en la disminución de la incidencia y prevalencia de esta enfermedad. Incentivamos el desarrollo de futuras investigaciones en este tema ya, que creemos firmemente es una opción que merece ser considerada para alcanzar una mejor salud.

\section{Bibliografía}

1. OMS. Inactividad física: un problema de salud pública mundial [Internet]. 2019 [cited 26 October 2019]. Available from: https://www.who.int/dietphysicalactivity/factsheet inactivity/es/.

2. Cristi-Montero C. Considerations regarding sedentary and physical inactivity. Aten Primaria 2016:48(5):341.

3. Dempsey P C, Dunstan D W, Larsen R, Lambert GW, Kingwell BA, Owen N. Prolonged uninterrupted sitting increases fatigue in type 2 diabetes. Diabetes Research and Clinical Practice 2018; 135:128-133.

4. Biswas A, Oh PI, Faulkner GE, Bajaj RR, Silver MA, Mitchell MS, Alter DA. Sedentary time and its association with risk for disease incidence, mortality, and hospitalization in adults. Ann Intern Med 2015;162(2):123. https://doi.org/10.7326/M14-1651.

5. Fazzi C, Saunders DH, Linton K, Norman JE, Reynolds RM. Sedentary behaviours during pregnancy: a systematic review. Int J Behav Nutr Phys Act 2017;14(1):32. https://doi.org/10.1186/s12966-017-0485-z.

6. Wagnild JM, Hinshaw K, Pollard T. Associations of sedentary time and selfreported television time during pregnancy with incident gestational diabetes and plasma glucose levels in women at risk of gestational diabetes in the UK. BMC Public Health 2019; 19:575. https:// doi.org/10.1186/s12889-019-6928-5 